\title{
AVANÇO DA COVID-19 NO ESTADO DA PARAÍBA E PERFIL DOS PACIENTES QUE FORAM A ÓBITO NOS PRIMEIROS QUARENTA E CINCO DIAS DE CASOS REGISTRADOS
}

\section{ADVANCEMENT OF COVID-19 IN THE STATE OF PARAIBA AND PROFILE OF PATIENTS WHO HAVE BEEN DEATH IN THE FIRST FORTY AND FIVE DAYS OF REGISTERED CASES.}

\author{
Martha Priscila Bezerra Pereira \\ Doutora em Geografia, UFCG \\ mpbcila@yahoo.com.br
}

Andréa Leandra Porto Sales Doutora em Geografia, UFPB andreaportosales@gmail.com

Xisto Serafim de Santana de Souza Júnior

Doutor em Geografia, UFCG xtojunio@yahoo.com.br

\begin{abstract}
RESUMO
A velocidade da expansão espaço-temporal da Covid-19 nos cinco continentes será considerado como marco da ruptura da atual lógica global/capitalista, haja vista as consequências socioeconômicas que serão deixadas em todos os países. No caso do Brasil a situação torna-se ainda mais complicada devido aos embates políticos entre o Governo Federal e os Governos Estaduais cujo resultado torna imprevisível qualquer precisão quanto ao controle da doença e redução da letalidade a exemplo dos casos evidenciados nos primeiros 45 dias de registros oficiais da Covid-19 no Estado da Paraíba. Desta forma, este artigo busca analisar o avanço da Covid-19 no estado da Paraíba e o perfil dos pacientes que foram a óbito nos primeiros quarenta e cinco dias de casos registrados. Para esta pesquisa foram realizadas as seguintes etapas: a) levantamento de referências; b) levantamento documental; c) levantamento de dados estatísticos; d) espacialização dos casos da Covid-19 no estado da Paraíba. Como resultados principais, percebeu-se que o avanço da Covid-19 no Estado da Paraíba ocorreu inicialmente seguindo o curso das principais rodovias do estado, chegando aos municípios de maior centralidade, e desses para municípios de menor; com exceção dos casos importados. Os dados de mortalidade apontam para uma maior vulnerabilidade de pessoas a partir de quarenta anos, se agravando para pacientes com mais de sessenta anos. Entre as comorbidades mais significativas que levam a óbito parece até o momento ser: a diabetes, a hipertensão e cardiopatias em geral. As fontes de dados, indicadores e variáveis estão disponíveis em vários endereços eletrônicos, sendo necessário o esforço da sistematização em trabalhos futuros.
\end{abstract}

Palavras-chave: Coronavirus. Covid-19. Paraíba. Perfil. Análise espacial.

\begin{abstract}
The speed of Covid-19's space-temporal expansion on the five continents will be considered as mark of the rupture of the current global/capitalist logic, considering the socioeconomic consequences that will be left in all countries. In the case of Brazil, the situation becomes even more complicated due to the political classes between the Federal Government and the State Governments, the result of which makes any precision regarding the control of the disease and reduction of lehality unpredictable, as in the cases evidenced in the first 45 days of registration. Covid-19 officers in the State of Paraiba. Thus, this article seeks to analyze the progress of Covid-19 in the state of Paraiba and the profile of patients who died in the first forty-five days of registered cases. For this research, the following steps were performed: a) survey of references; b) documentary survey; c) survey of statistical data; d) spatialization of Covid-19 cases in the state of Paraiba. As main results, it was noticed that the advance of Covid-19 in the State of Paraiba occurred initially following the course of the main highways of the state, arriving to municipalities of greater centrality, and of these for municipalities of smaller; with the exception of imported cases. Mortality data point to a greater vulnerability of
\end{abstract}

Recebido em: 10/05/2020

Aceito para publicação em: 17/05/2020. 
people over forty years old, worsening for patients over sixty years old. Among the most significant comorbidities that lead to death it seems so far to be: diabetes, hypertension and heart disease in general. The sources of data, indicators and variables are available at various electronic addresses, requiring systematization efforts in future works.

Key-words: Coronavirus. Covid-19. Paraiba. Profile. Spatial analysis.

\section{INTRODUÇÃO}

A COVID-19 faz parte de um grupo denominado Coronavírus que acomete a população humana esporadicamente (FREITAS, NAPIMOGA, DONALISIO, 2020; HOEK, PYRC, JEBBINK, 2004; SENHORAS, 2020; WOO, LAU, CHU, et al, 2005). De acordo com os registros oficiais, a Organização Mundial de Saúde foi informada em 31 de dezembro de 2019 que estava ocorrendo casos de novo Coronavírus na cidade de Wuhan, província de Hubei, China, sendo este classificado como uma pneumonia de etiologia desconhecida. Em 20 de janeiro de 2020 já havia 258 casos na China com três mortes. Também já havia casos importados (provenientes de Wuhan) na Tailândia, Japão e República da Coreia (WHO, 2020). Posteriormente foi especificado que a doença seria COVID-19.

Entre os debates que cercam as explicações da difusão do vírus compreendemos que o fator geográfico associado às diferentes formas de mobilidade espacial se caracteriza como relevantes nessa explicação. De fato, a interação espacial estabelecida entre os países através de diferentes formas de transportes influenciou na velocidade da difusão da Covid-19 segundo o sistema predominante de cada local. Desta forma, o vírus rapidamente atingiu vários países e continentes [EUA (23/01), Vietnã e Singapura (24/01), Austrália, Nepal e França (25/01), Malásia (26/01), Canadá (27/01), Camboja, Srilanka e Alemanha (28/01), Emirados Árabes (29/01), Filipinas, Índia e Finlândia (30/01), Itália (30/01), etc (WHO, 2020)], porém nos locais, a velocidade de contaminação seguiu cursos diferenciados, a depender das ações dos governos no tocante a medidas mitigadoras de controle e da conectividade entre os espaços de cada país (Figura 1).

Figura 1 - Número de casos de Coronavírus/Covid-19 por país e continente entre 17 de março e 06 de maio de 2020

\begin{tabular}{|c|c|c|c|c|c|c|c|c|c|c|c|c|c|}
\hline & & \multicolumn{2}{|l|}{$17 / \mathrm{mar}$} & \multicolumn{2}{|l|}{$27 / \mathrm{mar}$} & \multicolumn{2}{|l|}{$06 / a b r$} & \multicolumn{2}{|l|}{$16 / a b r$} & \multicolumn{2}{|l|}{$26 / a b r$} & \multicolumn{2}{|l|}{$06 / \mathrm{mai}$} \\
\hline 1 & 1.394 .550 .000 & China & 81.032 & Estados Unidos & 80.021 & Estados Unidos & 367.507 & Estados Unidos & 670.353 & Estados Unidos & 965.426 & Estados Unidos & 1.263 .092 \\
\hline 2 & 60.390 .560 & 0 Italia & 27.980 & China & 81.782 & Espanha & 136.675 & Espanha & 184.948 & Espanha & 226.629 & Espanha & 253.682 \\
\hline 3 & 82.207 .000 & 0 Irã & 14.991 & Italia & 80.589 & Italia & 132.547 & Italia & 168.941 & Italia & 197.675 & Italia & 214.457 \\
\hline 4 & 46.733 .038 & 3 Espanha & 9.428 & Espanha & 56.197 & França & 98.984 & França & 147.091 & Reino Unido & 154.037 & Reino Unido & 201.101 \\
\hline 5 & 51.811 .167 & Corea do Sul & 8.236 & Alemanha & 43.646 & Alemanha & 103.374 & Alemanha & 137.698 & França & 162.220 & França & 174.191 \\
\hline 6 & 82.979 .100 & Alemanha & 7.174 & França & 29.406 & Reino Unido & 52.279 & Reino Unido & 104.146 & Alemanha & 157.770 & Alemanha & 168.162 \\
\hline 7 & 66.992 .000 & França & 5.397 & Irã & 29.406 & China & 82.665 & Turquia & 74.193 & Russia & 80.949 & Russia & 165.929 \\
\hline 8 & 328.700 .000 & Estados Unidos & 4.138 & Suiça & 11.811 & Turquia & 30.217 & Irã & 77.995 & Turquia & 110.130 & Turquia & 131.744 \\
\hline 9 & 10.223 .505 & Suiça & 2.200 & Reino Unido & 11.772 & Irã & 60.500 & China & 82.367 & Brasil & 63.100 & Brasil & 126.611 \\
\hline 10 & 66.040 .229 & Reino Unido & 1.551 & Corea do Sul & 9.241 & Bélgica & 20.814 & Russia & 27.938 & Irã & 90.481 & Irã & 101.650 \\
\hline 11 & 17.298 .600 & Países Baixos & 1.414 & Países Baixos & 7.459 & Russia & 6.343 & Brasil & 30.683 & China & 82.827 & China & 82.883 \\
\hline 12 & 5.309 .659 & Noruega & 1.312 & Austria & 6.847 & Brasil & 12.183 & Bélgica & 34.809 & Canada & 47.143 & Canada & 63.496 \\
\hline 13 & 10.223 .505 & Suécia & 1.103 & Bélgica & 6.235 & Canada & 16.667 & Canada & 30.973 & Peru & 27.517 & Peru & 54.814 \\
\hline 14 & 11.454 .906 & Bélgica & 1.058 & Canada & 3.878 & Países Baixos & 18.926 & Paises Baixos & 29.383 & Bélgica & 46.134 & India & 52.987 \\
\hline 15 & 8.859 .992 & Austria & 1.018 & Turquia & 3.629 & Suíça & 21.657 & Suiça & 26.732 & India & 27.890 & Bélgica & 50.781 \\
\hline 16 & 5.806 .081 & Dinamarca & 932 & Portugal & 3.544 & Portugal & 11.730 & Portugal & 18.841 & Países Baixos & 38.040 & Paises Baixos & 41.319 \\
\hline 17 & 126.320 .000 & Japão & 825 & Noruega & 3.346 & India & 4.778 & India & 13.430 & Equador & 22.719 & Arabia Saudita & 31.938 \\
\hline 18 & 32.614 .200 & Malásia & 566 & Brasil & 2.915 & Irlanda & 5.364 & Peru & 12.491 & Arabia Saudita & 17.522 & Suiça & 30.060 \\
\hline 19 & 2.766 .459 & Catar & 439 & Suécia & 2.840 & Austria & 12.297 & Irlanda & 13.271 & Suiça & 29.061 & Equador & 29.420 \\
\hline 20 & 37.373 .000 & Canada & 405 & Australia & 2.810 & Peru & 2.561 & Suécia & 12.540 & Portugal & 23.864 & Mexico & 26.025 \\
\hline & Países do co & ontinente asiático & & $\begin{array}{l}\text { Fonte: Dado } \\
\text { Autoria:Sou }\end{array}$ & $\begin{array}{l}\text { disponive } \\
\text { a Júniore }\end{array}$ & $\begin{array}{l}\text { veis em: https://n } \\
\text { e Pereira, } 2020 .\end{array}$ & worldome & eters.info/corc & 15/country & $\mathrm{r} /$ /china//Acess & 08 de mai & io de 2020) & \\
\hline & Países do col & ntinente europeu & & & & & & & & & & & \\
\hline & ises do c & an & & & & & & & & & & & \\
\hline
\end{tabular}

Fonte: Worldmeters. Disponível em: https://worldmeters.info/country/china/. Acesso em 08 de maio de 2020. Organizado por Souza Júnior, XSS e Pereira, MPB (2020) 
A figura acima evidencia bem a relação do predomínio dos casos de Covid-19 entre os continentes. A partir de um intervalo temporal de 10 dias considerando uma semana após o reconhecimento da ONU de que a Covid-19 se tratava de pandemia até o dia 06 de maio observa-se uma tendência a redução dos casos significativos entre os países asiáticos com predomínio dos casos em países europeus e americanos. Isso se deve ao tipo de ação adotada (isolamento total ou parcial). Apesar de o transporte aéreo influenciar na contaminação a longa distância, fato que explicaria o aumento de casos em países da América, a figura 1 permite visualizar que os contatos por mobilidade terrestre entre os países europeus possibilitaram uma maior descentralização entre os países europeus resultando na intensificação de casos de contaminação. Essa constatação leva-nos a investigar quais dessas realidades corresponde aos estados brasileiros, em especial a Paraíba.

Entre os dez países com um maior número de casos de Covid-19 registrados oficialmente entre os cinco continentes, evidencia-se uma tendência na transferência do foco dos casos de Covid-19 da Ásia para a Europa, seguida para as Américas. Tal observação nos levou ao questionamento da reprodução dessa tendência no Brasil. Com a descentralização dos casos da região Sudeste para a região Nordeste, percebe-se uma dinâmica de disseminação que acompanha os fluxos das redes urbanas brasileiras. Ademais, o fato dos casos confirmados crescerem exponencialmente em segmentos da sociedade menos favorecidos tanto financeiramente quanto no atendimento junto ao serviço público de saúde, propõe-se a considerar a renda como uma variável importante na análise dos casos de Covid19 no Brasil e em especial no Estado da Paraíba, cujo PIB é um dos mais baixos do país e que nos últimos dias tem aumentado significativamente o número de casos de Covid-19.

Ao longo dos primeiros 45 dias a Paraíba foi o Estado com maior número de contaminados ficando atrás apenas do Ceará, Pernambuco, Maranhão e Bahia (Gráfico 1).

\section{Gráfico 1 - Nordeste: casos de Covid-19 por estados nos primeiros 45 dias desde a primeira ocorrência}

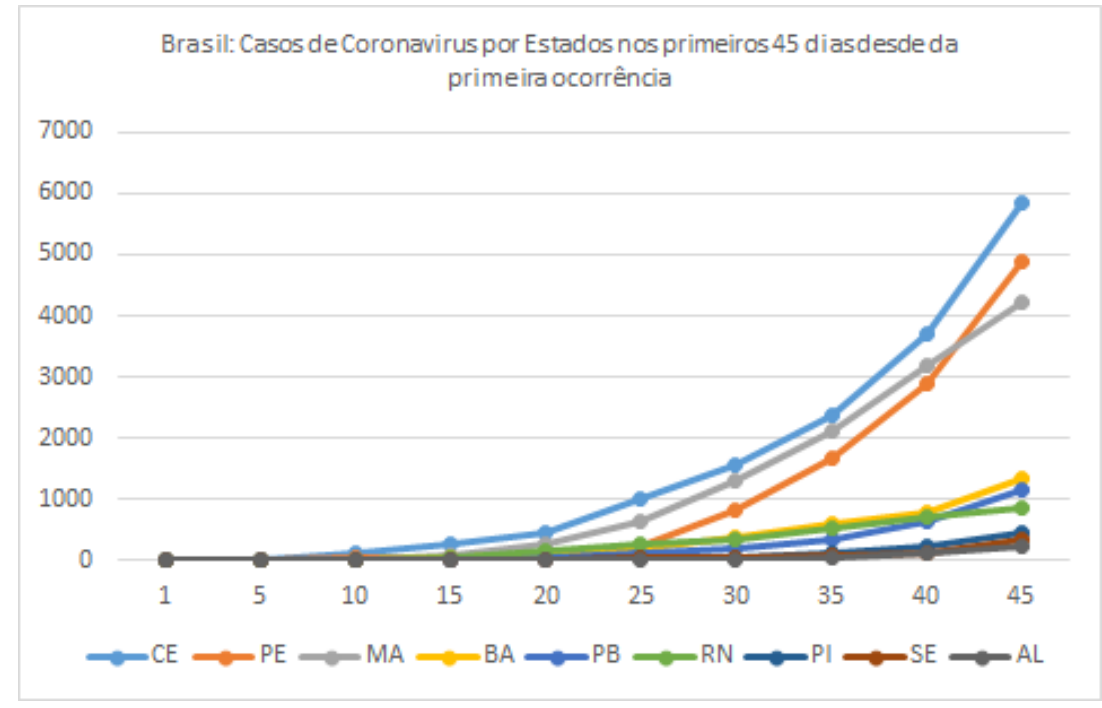

Fonte: Ministério da Saúde. Organizado por Souza Júnior, X.S.S. (2020)

Desta forma, este trabalho busca analisar o avanço da Covid-19 no estado da Paraíba e o perfil dos pacientes que foram a óbito nos primeiros quarenta e cinco dias de casos registrados, ou seja, entre 22 de março e 05 de maio de 2020.

Por não ser possível coletar dados suficientes sobre o perfil de pacientes, devido essas informações estarem disponíveis apenas até o dia 09 de abril (dia 19 da epidemia na Paraíba), optou-se pelo perfil dos pacientes que foram a óbito, pois, mesmo que haja algumas informações incompletas, foi realizada a descrição mínima caso a caso no período analisado.

Além da introdução e considerações finais o texto foi subdividido em cinco partes. Na primeira "Metodologia" foi realizada uma breve apresentação dos procedimentos executados. $\mathrm{Na}$ "Fundamentação Teórica" trabalhou-se com a Teoria da Difusão Espacial, associada às redes técnicas 
para tentar entender como ocorreu a expansão espacial da Covid-19, cujo alcance e velocidade levou a ser classificada pela Organização Mundial de Saúde (OMS) em 11 de março como pandemia.

Na parte "Avanço da Covid-19 no estado da Paraíba" foi observada a expansão da morbidade no Estado por quinzena e a quantidade de óbitos acumulados até o dia 05 de maio de 2020 (dia 45 da Covid-19 no Estado da Paraíba). A partir dessas informações fez-se uma breve análise.

No item "Perfil dos pacientes que vieram a óbito" foram trabalhadas todas as informações fornecidas pela SES-PB em relação aos pacientes que foram a óbito sendo possível ter informações sobre o gênero, idade, condição de risco, tempo de início dos sintomas até o óbito, tempo de hospitalização e o local do óbito.

Por último, na parte dos "Indicadores e variáveis importantes para correlacionar as informações obtidas" busca-se demonstrar que o manejo desses dados é apenas o início do trabalho no sentido de se entender possíveis rotas de difusão dentro da unidade da federação ou mesmo dentro de um município.

Este artigo surgiu a partir da discussão de um grupo de geógrafos denominado "Força Tarefa de Geógrafos" em que vários profissionais de vários estados do Brasil estão fazendo uma sistematização do que está ocorrendo em várias escalas geográficas.

\section{METODOLOGIA}

Para esta pesquisa foram realizadas as seguintes etapas: a) levantamento de referências; b) levantamento documental; c) levantamento de dados estatísticos; d) espacialização dos casos da COVID-19 no estado da Paraíba.

O levantamento de referências foi realizado nos portais do Google Acadêmico e Sciello, além de livros de autores relacionados à Geografia e à Saúde Coletiva.

O levantamento documental foi realizado através do site do Ministério da Saúde e da Organização Mundial de Saúde/ World Health Organization (OMS/ WHO).

O levantamento de dados estatísticos foi realizado junto à sala de imprensa da Secretaria Estadual da Paraíba.

A espacialização e discussão dos dados foram feitas com base nos dados oficiais disponibilizados pela Secretaria Estadual de Saúde do Estado da Paraíba.

\section{FUNDAMENTAÇÃO TEÓRICA}

A busca do entendimento da rápida difusão da Pandemia provocada pela Covid-19 pode ocorrer pela compreensão associativa da Teoria da Difusão Espacial (HAGERSTRAND, 1967) com a Teoria da Natureza do Espaço (SANTOS, 1997). A Teoria da Difusão Espacial trata da difusão de inovações como um processo espacial, para o qual a localização geográfica é um elemento importante na análise e não apenas um procedimento metodológico necessário. Para Torsten Hagerstrand (1967), a localização geográfica entendida como "the visible cultural landscape" oferece dados e informações importantes para a manifestação das inovações. A qual se pode considerar as doenças virais enquanto tal pelo fato da dependência do hospedeiro para contágio. Já a Teoria da Natureza do Espaço do Milton Santos aborda a determinação do estado das técnicas nas características da sociedade e do espaço geográfico.

Hagerstrand (1967) defende que os processos de difusão não aparecem imediatamente sobre toda a superfície terrestre, algumas pessoas e alguns lugares tem acesso imediato às inovações, outros depois e alguns pelas condições culturais, ou como aqui irá ser tratado pela técnica, jamais terão acesso. A difusão possui pelo menos três padrões: difusão em $\mathbf{S}$ na qual há a difusão de determinada inovação [no nosso caso de uma doença contagiosa] até o momento da saturação; a difusão hierárquica, cujos lugares centrais hierarquicamente superiores tendem a ser contemplados inicialmente [no nosso caso, as cidades mais centrais tendem a sofrer mais rápido com a difusão da COVID-19] e; o padrão de contágio, em que inicialmente ocorre o fenômeno em seu espaço imediato, e pelo contato atinge áreas mais distantes (figura 2) (SILVA, 1995).

Se pensarmos a nível mundial, ou mesmo em determinado município, há uma difusão em $\mathrm{S}$, em que a Covid-19 atinge o número máximo de pessoas até sua saturação (neste caso se aplica a explicação do achatamento da curva para não colapsar o sistema de saúde com uma demanda maior de leitos de UTI e respiradores - quanto mais devagar as pessoas adoecerem, mais o sistema tem condições de atender). 
Figura 2 - Padrões de difusão espacial segundo Torsten e Hagestrand

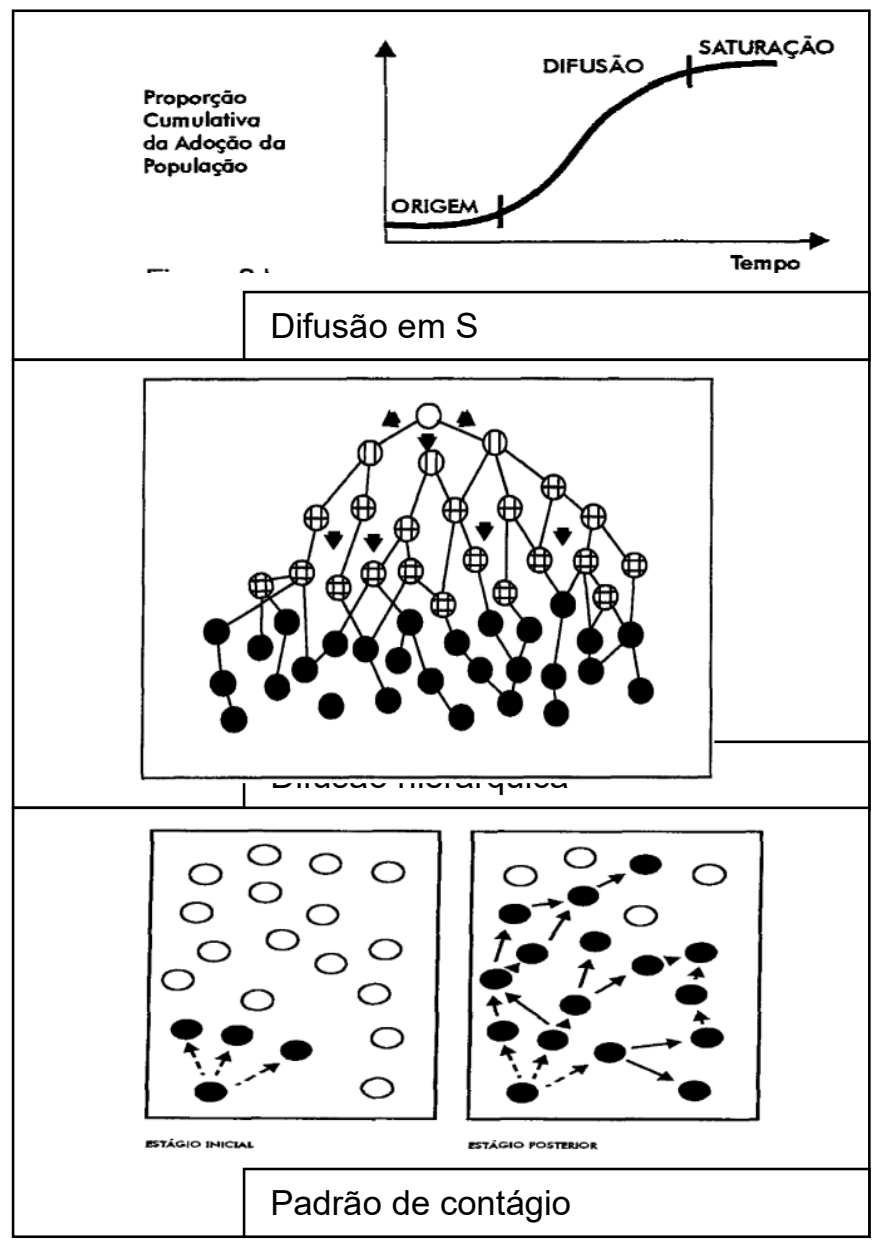

Se houver a necessidade de se pensar regionalmente, essa difusão ocorre hierarquicamente no território (das cidades mais centrais para as cidades menores e sofre interferência direta das redes técnicas - neste caso se aplica como medida sanitária o bloqueio sanitário dessas redes, seja aeroviária, rodoviária, ferroviária, fluvial, etc).

E no cotidiano, essa difusão ocorre por contato, no padrão de contágio, sendo tão necessário tomar as medidas de precaução pessoal apresentadas pela OMS e pelo Ministério da Saúde.

\section{AVANÇO DA COVID-19 NO ESTADO DA PARAÍBA}

No que diz respeito ao avanço da Covid-19, na primeira quinzena atingiu a maioria das cidades de maior porte do Estado da Paraíba (João Pessoa, Campina Grande, Sousa), com casos importados em Junco do Seridó e Igaracy (cidades de menor porte, mas que também teve casos).

$\mathrm{Na}$ segunda quinzena se difunde para as cidades próximas às principais cidades, todavia, continua o padrão hierárquico de dispersão para as cidades de porte médio (neste caso as cidades consideradas sede de região imediata)

$\mathrm{Na}$ terceira quinzena começou a atingir em sua maioria os municípios do entorno, ocorrendo uma difusão tanto hierárquica quanto de contágio, pois seria uma situação mais local, de contato pessoal (Mapa 01). 
Avanço da Covid-19 no estado da Paraíba e perfil dos pacientes que foram a óbito nos primeiros quarenta e cinco dias de casos registrados
Martha Priscila Bezerra Pereira

Andréa Leandra Porto Sales Xisto Serafim de Santana de Souza Júnior

Mapa 1 - Avanço da Covid-a9 no estado da Paraíba: primeiros 45 dias (22/03 a 05/05/2020)

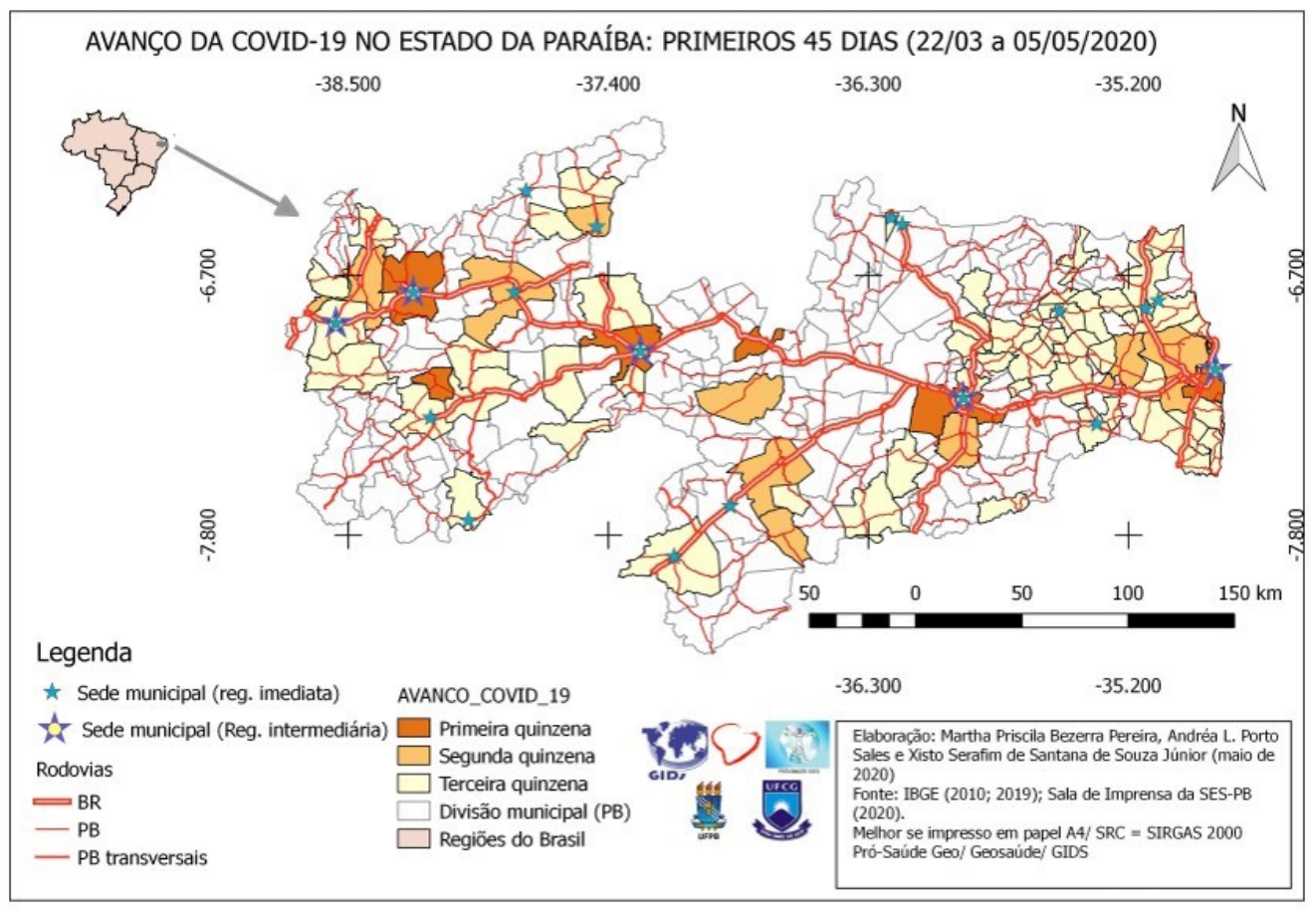

Em relação aos óbitos, percebe-se que este se concentra principalmente na região metropolitana de João Pessoa (especialmente João Pessoa e Santa Rita), onde estão concentrados os maiores números de casos. No quadragésimo quinto dia João Pessoa concentrava 865 casos, enquanto Santa Rita, 129 casos (mapa 2).

Em termos de municípios atingidos, o estado da Paraíba possui 223 municípios, destes, $73(32,7 \%)$ já tiveram casos notificados de COVID e $22(9,86 \%)$ já registraram pelo menos 1 óbito.

Mapa 2 - Covid-19: casos confirmados e óbitos na Paraíba em 05 de maio de 2020.

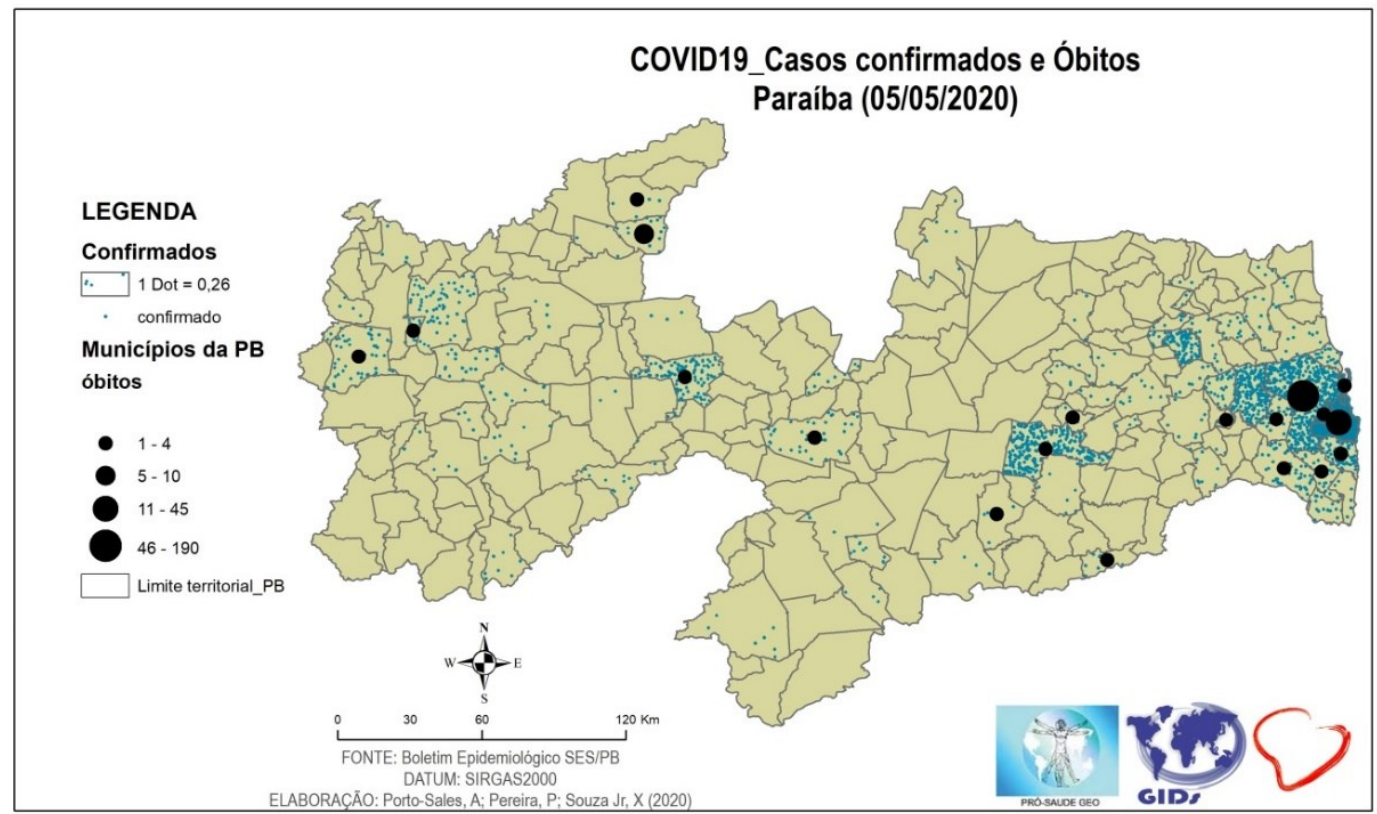




\section{PERFIL DOS PACIENTES QUE FORAM A ÓBITO}

Nessa parte serão consideradas as informações fornecidas pela Secretaria do Estado da Paraíba quanto aos óbitos. As informações fornecidas foram: gênero, idade, município de moradia, se havia comorbidade, tempo de início do sintoma até o óbito e se foi atendido em hospital público ou privado. Ao final, será realizada uma breve comparação da situação do estado com o Brasil.

Quanto ao gênero, a maioria estava representada pelo gênero masculino (60 indivíduos $-64,52 \%$ ), as mulheres representaram $35,48 \%$ (33 mulheres). Sendo o universo $93^{2}$ indivíduos que foram a óbito em 45 dias no estado da Paraíba.

No que diz respeito à faixa etária, a idade do óbito variou de 4 meses até 95 anos, começando a ficar preocupante a partir da faixa etária dos quarenta anos. Todavia, a faixa etária a partir dos sessenta anos (60 a 95 anos) teve a maior representatividade (quadro 1).

Quadro 1 - Faixa etária dos indivíduos que foram a óbito por Covid-19

\begin{tabular}{|c|c|c|c|c|}
\hline \multirow{2}{*}{ FAIXA ETÁRIA } & \multicolumn{4}{|c|}{ GÊNERO } \\
\cline { 2 - 5 } & \multicolumn{2}{|c|}{ HOMEM } & QUANT & $\%$ \\
\cline { 2 - 5 } & QUANT & $\%$ & 1 & 3,03 \\
\hline Criança - 0 a 9 anos & 0 & 0 & 0 & 0 \\
\hline Adolescente - 10 a 19 anos & 0 & 0 & 2 & 6,06 \\
\hline Jovem - 20 a 34 anos & 1 & 1,67 & 7 & 21,21 \\
\hline Adulto - 35 a 59 anos & 26 & 43,33 & 23 & 69,70 \\
\hline Idoso - 60 anos ou mais & 33 & 55 & 33 & 100 \\
\hline TOTAL & 60 & 100 & \multicolumn{2}{c|}{ MULHER } \\
\hline
\end{tabular}

Fonte: Sala de imprensa da Secretaria Estadual de Saúde da Paraíba. Disponível em: paraiba.pb.gov.br/diretas/saúde/coronavirus/noticias. Acesso entre 17 de março e 07 de maio de 2020. Organizado por Pereira, MPB (2020)

Foram várias as condições de risco associadas aos 93 pacientes, destas, a hipertensão com 22 pacientes $(23,65 \%)$, a diabetes com 21 pacientes $(22,58 \%)$ e cardiopatias com 20 pacientes $(21,5 \%)$ foram as que se destacaram (quadro 2).

Dos pacientes em que houve informação sobre o período do início dos sintomas até o óbito, houve uma média de 10,7 dias, variando de 1 a 30 dias em seus casos específicos.

Em relação às informações fornecidas, o período de internação variou de 1 a 15 dias, sendo a média de tempo de internação 4,7 dias.

No que diz respeito ao local de internação e, finalmente, o óbito, dos 93 pacientes, $62(66,67 \%)$ ocuparam hospitais públicos, $16(17,2 \%)$ ocuparam hospitais privados, os outros foram atendidos em UPA $(6,45 \%)$, faleceram na residência $(4,3 \%)$ ou não foi informado $(5,37 \%)$.

Dos que faleceram na residência, dois residiam em Santa Rita, um em João Pessoa (próximo a hospitais de referência para COVID-19) e um morava em Mari. Dos quatro pacientes, três tinham problemas psiquiátricos ou neurológicos.

Para finalizar este item, fazendo uma breve análise do estado da Paraíba em relação ao Brasil quanto aos coeficientes de letalidade, de mortalidade por causa, e das taxas de incidência e prevalência podese entender um pouco de como está a situação da Paraíba em relação ao Brasil a partir de seus dados oficiais.

Relacionando o estado da Paraíba (entre 22/03 e 05/05/20) com o Brasil (27/02 a 11/04/20) pode-se perceber, a partir da análise entre o primeiro e o quadragésimo quinto dia que a situação da Paraíba parece estar pior do que a média geral do Brasil.

${ }^{2}$ A partir do dia 29 de abril a informação do total de óbitos está acrescentada um número a mais devido na apresentação do perfil do paciente que foi a óbito haver um indivíduo a mais. 
Avanço da Covid-19 no estado da Paraíba e perfil dos pacientes que foram a óbito nos primeiros quarenta e cinco dias de casos registrados
Martha Priscila Bezerra Pereira

Andréa Leandra Porto Sales Xisto Serafim de Santana de Souza Júnior

Quadro 2 - Síntese por município dos pacientes que foram à óbito por Covid-19

\begin{tabular}{|c|c|c|c|c|c|c|c|}
\hline MUNICÍPIO & $\begin{array}{c}\text { GÊNERO } \\
\text { (H=HOMEM;M } \\
\text { =MULHER) }\end{array}$ & $\begin{array}{c}\text { FAIXA } \\
\text { ETÁRIA }\end{array}$ & $\begin{array}{l}\text { N_COND } \\
\text {. RISCO }\end{array}$ & TIPO RISCO & $\begin{array}{l}\text { DIAS } \\
\text { SINT } \\
\text { MED }\end{array}$ & $\begin{array}{l}\text { DIAS } \\
\text { INTERN } \\
\text { MED }\end{array}$ & $\begin{array}{l}\text { LOCAL } \\
\text { OBITO }\end{array}$ \\
\hline Alhandra & $1 \mathrm{~h}, 1 \mathrm{~m}$. & $2(60+)$ & 2 & $\begin{array}{l}1 \text { cardiopatia, } 1 \\
\text { diabetes, } 1 \text { doença } \\
\text { neurológica, } \\
\text { hipertensão, } 1 \text { doença } \\
\text { renal, obesidade, } \\
\text { sequela } \mathrm{AVC}^{3}\end{array}$ & 7 & $\mathrm{NI}^{4}$ & $\begin{array}{l}2 \\
\text { Hospital } \\
\text { público }\end{array}$ \\
\hline Bayeux & $3 \mathrm{~h}$ & $\begin{array}{l}1(35-59) ; 2 \\
(60+)\end{array}$ & 3 & $\begin{array}{ll}\text { Etilismo, } & 2 \\
\text { Hipertensão, } & 2 \\
\text { tabagismo, } & \end{array}$ & 9 & 4 & $\begin{array}{l}2 \\
\text { Hospital } \\
\text { público e } \\
1 \\
\text { UPA em }\end{array}$ \\
\hline Boqueirão & $1 \mathrm{~h}$ & $1(35-59)$ & 1 & 1 tabagismo & $\mathrm{NI}$ & $\mathrm{NI}$ & 1 UPA \\
\hline $\begin{array}{l}\text { Brejo do } \\
\text { Cruz }\end{array}$ & $2 \mathrm{~h}$ & $2(60+)$ & 2 & $\begin{array}{l}\text { Doença renal, }{ }^{2} \\
\text { DPOC }^{5}, 2 \text { hipertensão, }\end{array}$ & $\mathrm{NI}$ & 3 & $\begin{array}{l}2 \\
\text { Hospital } \\
\text { Público }\end{array}$ \\
\hline Cabedelo & $3 \mathrm{he} 1 \mathrm{~m}$ & $\begin{array}{l}1(35-59) ; 3 \\
(60+)\end{array}$ & 2 & $\begin{array}{lll}2 & \text { Cardiopatia, } & 1 \\
\text { diabetes } & \end{array}$ & 11 & $\mathrm{NI}$ & $\begin{array}{l}3 \\
\text { Hospital } \\
\text { público, } 1 \\
\text { Hospital } \\
\text { privado. }\end{array}$ \\
\hline Cajazeiras & $1 \mathrm{~h}$ & $1(60+)$ & 1 & 1 Hipertensão & 10 & $\mathrm{NI}$ & $\begin{array}{l}1 \\
\text { Hospital } \\
\text { público }\end{array}$ \\
\hline $\begin{array}{l}\text { Campina } \\
\text { Grande }\end{array}$ & $1 \mathrm{he} 1 \mathrm{~m}$ & $\begin{array}{l}1(35-59) ; 1 \\
(60+)\end{array}$ & 1 & 1 Neoplasia & $\mathrm{NI}$ & 8 & $\begin{array}{l}1 \text { UPA, } 1 \\
\text { Hospital } \\
\text { privado. }\end{array}$ \\
\hline Conde & $1 \mathrm{~h}$ & 1 (35-59) & $\mathrm{NI}$ & $\mathrm{NI}$ & $\mathrm{NI}$ & $\mathrm{NI}$ & $\begin{array}{l}1 \\
\text { Hospital } \\
\text { público }\end{array}$ \\
\hline $\begin{array}{ll}\text { Cruz do } \\
\text { Espírito } \\
\text { Santo }\end{array}$ & $1 \mathrm{~h}$ & $1(60+)$ & 1 & 1 Doença renal & 5 & $\mathrm{NI}$ & $\begin{array}{l}1 \\
\text { Hospital } \\
\text { público }\end{array}$ \\
\hline $\begin{array}{l}\text { João } \\
\text { Pessoa }\end{array}$ & $25 \mathrm{~h} \mathrm{e} 19 \mathrm{~m}$ & $\begin{array}{ll}3 & (20-34) ; \\
14 & (35-59) ; \\
27 & (60+)\end{array}$ & 31 & $\begin{array}{lr}1 \text { Anemia, } 1 \text { asma, } 10 \\
\text { Cardiopatia, } 1 \text { Def. } \\
\text { intelectual, } 5 \text { Diabetes, } \\
2 \text { doença neurológica, } \\
\text { doença pulmonar, } \\
\text { doença respiratória, } \\
\text { etillista, } 1 \text { gestante, } \\
\text { Hipertensão, } \\
\text { neoplasia, } 1 \text { obesidade, } \\
1 \text { puérpera, } 1 \\
\text { tabagismo, }\end{array}$ & 11 & 9,5 & $\begin{array}{l}14 \text { Hosp. } \\
\text { Privado, } \\
25 \text { hosp. } \\
\text { Público, } \\
1 \text { em } \\
\text { residênci } \\
\text { a, } 4 \text { não } \\
\text { informad } \\
\text { o. }\end{array}$ \\
\hline $\begin{array}{l}\text { Junco do } \\
\text { Seridó }\end{array}$ & $1 \mathrm{~m}$ & $1(60+)$ & 1 & $\begin{array}{l}1 \text { Diabetes, } 1 \text { glaucoma, } \\
1 \text { meningogioma }\end{array}$ & 7 & 1 & $\begin{array}{l}1 \\
\text { Hospital } \\
\text { público }\end{array}$ \\
\hline Lagoa Seca & $1 \mathrm{~h}$ & $1(35-59)$ & 1 & 1 Etilista & 16 & $\mathrm{NI}$ & $\begin{array}{l}1 \\
\text { Hospital } \\
\text { público }\end{array}$ \\
\hline
\end{tabular}

\footnotetext{
${ }^{3} \mathrm{AVC}=$ Acidente Vascular Cerebral

${ }^{4} \mathrm{NI}=$ não informado

${ }^{5}$ DPOC= Doença Pulmonar Obstrutiva Crônica.

DOl:http://dx.doi.org/10.14393/Hygeia0054641
} 
Avanço da Covid-19 no estado da Paraíba e perfil dos pacientes que foram a óbito nos primeiros quarenta e cinco dias de casos registrados
Martha Priscila Bezerra Pereira

Andréa Leandra Porto Sales Xisto Serafim de Santana de Souza Júnior

Quadro 2 (cont.) - Síntese por município dos pacientes que foram à óbito por Covid-19

\begin{tabular}{|c|c|c|c|c|c|c|c|}
\hline MUNICÍPIO & $\begin{array}{c}\text { GÊNERO } \\
(\text { H=HOMEM; } \\
\text { M=MULHER) }\end{array}$ & $\begin{array}{l}\text { FAIXA } \\
\text { ETÁRIA }\end{array}$ & $\begin{array}{l}\text { N_CON } \\
\text { D. } \\
\text { RISCO }\end{array}$ & TIPO RISCO & $\begin{array}{l}\text { DIAS } \\
\text { SINT } \\
\text { MED }\end{array}$ & $\begin{array}{l}\text { DIAS } \\
\text { INTERN } \\
\text { MED } \\
\end{array}$ & $\begin{array}{l}\text { LOCAL } \\
\text { OBITO }\end{array}$ \\
\hline Mari & $1 \mathrm{~m}$ & $1(35-59)$ & 1 & $\begin{array}{l}1 \\
\text { psiquiátrico, } \\
\text { Obesidade }\end{array}$ & $\mathrm{NI}$ & $\mathrm{NI}$ & $\begin{array}{l}1 \\
\text { Residên } \\
\text { cia }\end{array}$ \\
\hline Marizópolis & $1 \mathrm{~m}$ & $1(60+)$ & 1 & 1 Hipertensão & $\mathrm{NI}$ & 1 & $\begin{array}{l}1 \\
\text { Hospital } \\
\text { público }\end{array}$ \\
\hline Patos & $2 \mathrm{he} 1 \mathrm{~m}$ & $3(35-59)$ & 3 & $\begin{array}{ll}2 \quad \text { Diabetes, } & 1 \\
\text { neoplasia, } & 1 \\
\text { hipertensão, } & 1 \\
\text { obesidade, } & \end{array}$ & $\mathrm{NI}$ & 5 & $\begin{array}{l}3 \\
\text { Hospital } \\
\text { público }\end{array}$ \\
\hline $\begin{array}{l}\text { Pedras de } \\
\text { Fogo }\end{array}$ & $1 \mathrm{~m}$ & $1(60+)$ & 1 & $\begin{array}{l}\text { diabetes, } 1 \text { doença } \\
\text { vascular crônica, } \\
\text { hipertensão, } \\
\text { obesidade }\end{array}$ & 8 & $\mathrm{NI}$ & $\begin{array}{l}1 \\
\text { Hospital } \\
\text { público }\end{array}$ \\
\hline $\begin{array}{l}\text { Riachão do } \\
\text { Poço }\end{array}$ & $1 \mathrm{~h}$ & $1(35-59)$ & 1 & $\begin{array}{ll}1 \quad \text { diabetes, } & 1 \\
\text { hipertensão, } & 1 \\
\text { problema renal } & \end{array}$ & $\mathrm{NI}$ & $\mathrm{NI}$ & $\begin{array}{l}1 \\
\text { Hospital } \\
\text { público }\end{array}$ \\
\hline Santa Rita & $15 \mathrm{~h} \mathrm{e} 4 \mathrm{~m}$ & $\begin{array}{l}8(35-59) \\
11(60+)\end{array}$ & 17 & 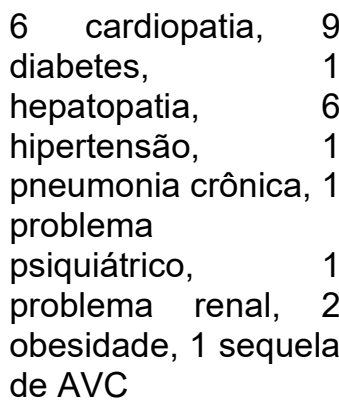 & $\begin{array}{l}13,1 \\
1\end{array}$ & 5,67 & $\begin{array}{l}3 \text { UPA, } \\
13 \\
\text { Hospital } \\
\text { público, } \\
2 \\
\text { residênc } \\
\text { ia, } 1 \mathrm{NI} .\end{array}$ \\
\hline São Bento & $1 \mathrm{~h}$ & $1(60+)$ & 0 & - & 12 & $\mathrm{NI}$ & $\begin{array}{l}1 \\
\text { Hospital } \\
\text { público }\end{array}$ \\
\hline Sapé & $1 \mathrm{~m}$ & $1(60+)$ & 1 & $\begin{array}{l}1 \text { diabetes, } \\
\text { hipertensão, } \\
\text { problema renal, } \\
\text { sequela de AVC }\end{array}$ & $\mathrm{NI}$ & $\mathrm{NI}$ & $\begin{array}{l}1 \\
\text { Hospital } \\
\text { Privado. }\end{array}$ \\
\hline Taperoá & $1 \mathrm{~m}$ & $1(0-9)$ & 0 & - & $\mathrm{NI}$ & $\mathrm{NI}$ & $\begin{array}{l}1 \\
\text { Hospital } \\
\text { público }\end{array}$ \\
\hline Umbuzeiro & $1 \mathrm{~h}$ & $1(60+)$ & 1 & $\begin{array}{ll}1 \quad \text { cardiopatia, } & 1 \\
\text { hipertensão, } & 1 \\
\text { sequela de AVC } & \end{array}$ & 1 & 1 & $\begin{array}{l}1 \\
\text { Hospital } \\
\text { público }\end{array}$ \\
\hline
\end{tabular}

Fonte: Sala de imprensa da Secretaria Estadual de Saúde da Paraíba. Acesso entre 17 de março e 07 de maio de 2020 (PARAÍBA, 2020). Nas colunas dos dias de sintomas referidos e dias de internação médica foram colocados os valores médios dos pacientes que tiveram essa informação divulgada. Organizado por Pereira, MPB (2020) 
A taxa de prevalência (números de casos velhos + novos/ população exposta na área $x$ 100.000) no quadragésimo quinto dia estava na Paraíba em 37,16 casos por 100.000 habitantes, enquanto no Brasil em 9,97 casos por 100.000 habitantes. Pelo menos em termos de predição, pode-se deduzir que na Paraíba tem-se uma proporção maior da população acometida pelo Covid-19 neste período de 45 dias, sendo possível o término desse período ser abreviado, por outro lado, se não houver recursos humanos, leitos e respiradores suficientes para atender os casos mais graves, teremos posteriormente uma maior mortalidade proporcionalmente.

A taxa de incidência (número de casos novos/ população exposta $\times 100.000$ ) teve seu maior pico no quadragésimo quarto dia (3,53 novos casos para cada 100.000 habitantes) enquanto o Brasil neste mesmo período teve sua maior taxa de incidência no quadragésimo segundo dia, chegando a 1,02 casos para cada 100.000 habitantes. Apesar de tanto no Brasil quanto no estado da Paraíba a tendência é que esses números aumentem ainda por um tempo devido a Pandemia não ter chegado no seu nível máximo, ou no nível de saturação, o ritmo do estado tem sido um pouco mais acelerado, sendo potencialmente causador de mais óbitos proporcionalmente, tanto devido à velocidade como devido a outros fatores como um serviço de saúde eficiente em todos os municípios.

No que diz respeito ao coeficiente de letalidade (número de óbitos/número de casos x 100) tem sido muito maior no estado da Paraíba do que no Brasil. Como exemplo, pode-se citar que o maior resultado na Paraíba ocorreu no vigésimo quinto dia (14,55 óbitos para cada 100 casos), enquanto no Brasil, o maior resultado ocorreu no quadragésimo quinto dia (5,44 óbitos para cada 100 pessoas). Percebe-se que a letalidade está maior na Paraíba e está ocorrendo de maneira mais rápida do que na média para o Brasil. Ou seja, das pessoas que adoecem, uma proporção maior de pessoas vai a óbito, ou a quantidade de testes está sendo tão insuficiente que só estão testando os casos mais graves e que, na maioria, vai a óbito.

Quanto ao coeficiente de mortalidade por COVID-19 (número de óbitos por causa pela população equivalente na área $x$ 100.000), no quadragésimo quinto dia a Paraíba estava com 2,31 óbitos a cada 100.000 habitantes, enquanto no Brasil, no dia equivalente $\left(45^{\circ}\right)$ estava com um coeficiente de 0,54 óbitos para cada 100.000 habitantes. Essa informação demonstra que em relação a esta causa de morte, ao final desta Pandemia certamente haverá proporcionalmente mais óbitos do que para a média do Brasil.

\section{INDICADORES E VARIÁVEIS IMPORTANTES PARA CORRELACIONAR ÀS INFORMAÇÕES OBTIDAS}

A partir dos resultados deste texto podem-se mostrar algumas possibilidades de indicadores e variáveis importantes para se observar no município que apontam para a população mais vulnerável. Dentre esses indicadores estão as morbidades de maior importância (hipertensão, cardiopatia e diabetes), os indivíduos com idade acima de quarenta anos, se agravando para os pacientes a partir de sessenta anos.

Além destes, há a possibilidade da utilização de muitas outras informações que estariam modificando o comportamento da pandemia em localidades específicas (quadro 3 ). 
Quadro 3 - Variáveis e indicadores que podem interferir na velocidade de difusão da Covid-19 e outras doenças contagiosas

\begin{tabular}{|c|c|}
\hline VARIÁVEIS E INDICADORES & \multirow{6}{*}{ 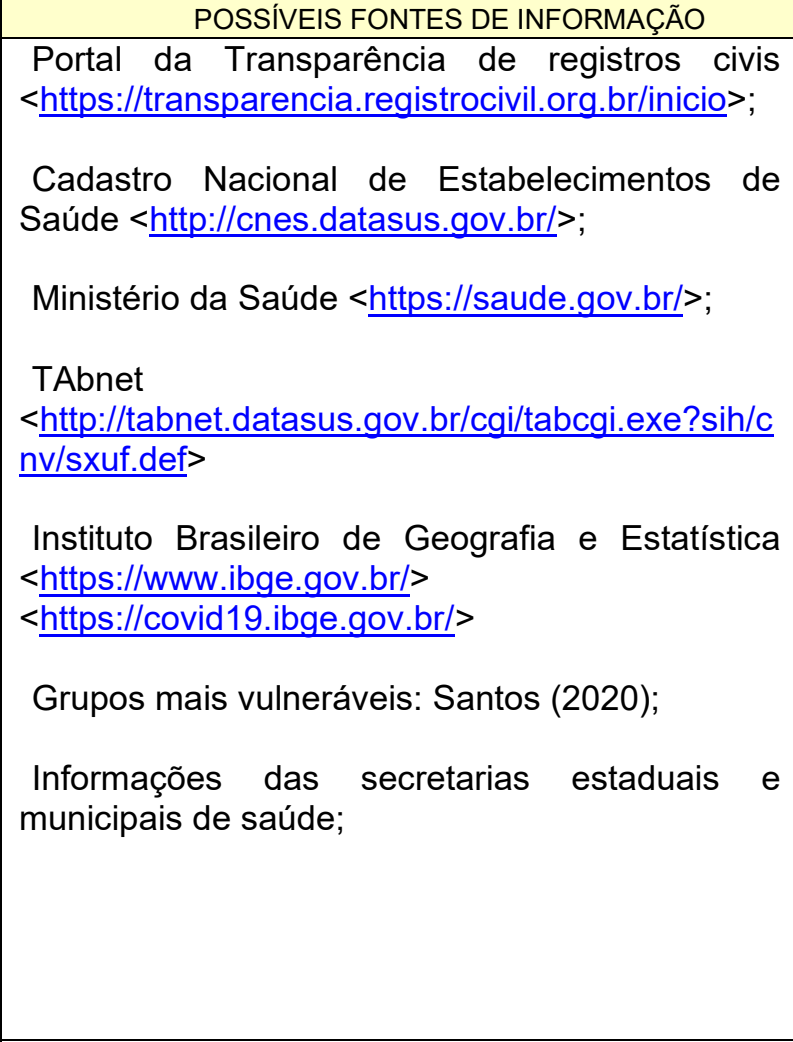 } \\
\hline $\begin{array}{l}\text { Sistema de Saúde: capacidade da infra-estrutura } \\
\text { em cuidar das pessoas (medidas de isolamento } \\
\text { social) }\end{array}$ & \\
\hline $\begin{array}{l}\text { - Identificação e localização dos grupos mais } \\
\text { vulneráveis (faixa etária, gênero, tipo de moradia, } \\
\text { quantidade de pessoas por domicílio, tipos de } \\
\text { ocupação, condição de saúde, renda, condição de } \\
\text { cidadania dentro da sociedade; comorbidades } \\
\text { que mais influenciam no óbito, grupos étnicos); } \\
\text { - Acompanhamento do número de casos em } \\
\text { determinado tempo em cada local; }\end{array}$ & \\
\hline $\begin{array}{l}\text { - Acompanhamento do número de casos de óbito } \\
\text { em determinado local; } \\
\text { - Acompanhamento das medidas sanitárias de } \\
\text { isolamento de cada local; }\end{array}$ & \\
\hline $\begin{array}{l}\text { - Levantamento da infra-estrutura disponibilizada } \\
\text { em cada local (número de leitos de UTI, } \\
\text { respiradores, equipamentos de uso individual }\end{array}$ & \\
\hline $\begin{array}{l}\text { para profissionais de saúde, médicos disponíveis, } \\
\text { enfermeiros disponíveis, ambulâncias equipadas } \\
\text { com respiradores, kits de testes, laboratórios } \\
\text { credenciados e capacidade de realização de } \\
\text { exames); } \\
\text { - Densidade demográfica; } \\
\text { - IDH_M. }\end{array}$ & \\
\hline $\begin{array}{l}\text { Redes técnicas: difusão hierárquica (barreiras } \\
\text { sanitárias) }\end{array}$ & \multirow{2}{*}{$\begin{array}{l}\text { Ministério da Infraestrutura } \\
\text { <https://www.infraestrutura.gov.br/>; } \\
\text { Santos (2020). }\end{array}$} \\
\hline $\begin{array}{l}\text { - Eixo de dispersão do vírus; } \\
\text { - Redes técnicas de dispersão do vírus; } \\
\text { - Redes técnicas que auxiliam nas medidas } \\
\text { sanitárias (saneamento ambiental); } \\
\text { - Distribuição das ambulâncias de forma a realizar } \\
\text { a comunicação entre municípios } \\
\text { hierarquicamente diferentes; } \\
\text { - Medidas relacionadas a barreiras sanitárias em } \\
\text { locais estratégicos na hierarquização dos } \\
\text { territórios. }\end{array}$ & \\
\hline $\begin{array}{l}\text { Objetos instalados em cada município e } \\
\text { recomendações de higiene (cuidados pessoais) }\end{array}$ & \multirow{4}{*}{$\begin{array}{l}\text { Fotos de noticiários; } \\
\text { Observação da paisagem de cada localidade; } \\
\text { Acompanhamento de vídeos e fotos em redes } \\
\text { sociais; } \\
\text { Gestão municipal de trânsito. }\end{array}$} \\
\hline - Medidas sanitárias individuais; & \\
\hline $\begin{array}{l}\text { - Acompanhamento de instalação de objetos e de } \\
\text { ações das pessoas; }\end{array}$ & \\
\hline $\begin{array}{l}\text { - Redes técnicas cotidianas dentro do município } \\
\text { (ônibus, trens urbanos, metrôs, mototaxi, uber, } \\
\text { etc.) }\end{array}$ & \\
\hline
\end{tabular}

Fonte: Discussão no grupo "Força tarefa de Geógrafos"; Informações fornecidas pelos portais oficiais; reportagens diversas, palestras. Observação realizada entre 18 de março e 08 de maio de 2020. Organizado por Pereira, MPB (2020).

Essas informações podem demonstrar os locais mais e menos vulneráveis para a pandemia de Covid19 assim como são apropriadas para outras epidemias ou pandemias que possam surgir. 


\section{CONSIDERAÇÕES FINAIS}

Percebeu-se que o avanço da Covid-19 de fato ocorreu inicialmente pelas principais rodovias do estado chegando aos principais municípios, e destes locais para municípios de menor porte, com exceção dos casos importados.

Os dados de mortalidade apontam para uma maior vulnerabilidade para pessoas a partir de 40 anos, se agravando para pacientes com mais de 60 anos, assim como as comorbidades mais significativas que levam a óbito parecem ser até o momento a diabetes, a hipertensão e cardiopatias em geral.

As fontes de dados, os indicadores e variáveis estão disponíveis em vários endereços eletrônicos, sendo necessário o esforço da sistematização em trabalhos posteriores.

As informações apresentadas neste texto são uma interpretação das informações oficiais sobre o estado da Paraíba, que serve como uma inferência do que de fato está ocorrendo, pois há indícios no cotidiano de reportagens e das redes sociais que demonstram haver muita subnotificação (seja por falta de kits para teste e/ou devido os testes realizados fora do âmbito de laboratórios credenciados ainda não serem aceitos para a notificação), muitos óbitos não estarem sendo associados à Covid-19, entre outras que não é objeto deste artigo.

\section{REFERÊNCIAS}

FREITAS, André Ricardo Ribas; NAPIMOGA, Marcelo; DONALISIO, Maria Rita. Análise da gravidade da pandemia de COVID-19. Epidemiologia e Serviços de Saúde. Brasília - DF. Secretaria de Vigilância em Saúde, v. 29, n.2, p. 1-5, 2020. Disponívem em: www.scielosp.org/pdf/ress/2020.v29n2/e2020119/pt. Acesso em 04 de maio de 2020. https://doi.org/10.5123/S1679-49742020000200008

HÄGESTRAND, Torsten. Innovation diffusion as a spatial process. Chicago: University of Chicago Press. Translation and postscript by Allan Pred, 1967.

HOEK, Lia van der; PYRC, Krzysztof; JEBBINK, Maarten F. et al. Identification of a new human coronavirus. Nature Medicine, vol. 10, n.4, april 2004. Disponível em: http://www.nature.com/naturemendicine. Acesso em 02 mai 2020. https://doi.org/10.1038/nm1024

SENHORAS, Elói Martins. Coronavírus e o papel das pandemias na história humana. Boca: Boletim de Conjuntura, vol. 1, n. 1, 2020. Disponível em: https://revista.ufrr.br/boca/article/view/Eloi/2899. Acesso em04 mai 2020.

SANTOS, Boaventura de Sousa. A cruel pedagogia do vírus. Coimbra: Almedina, 2020, 32p.

SANTOS, Milton. A natureza do espaço: técnica e tempo, razão e emoção. 2.ed. São Paulo - SP: Hucitec, 1997, 273p.

SILVA, Carlos Alberto Franco da. Os avatares da teoria da difusão espacial: uma revisão teórica. Revista Brasileira de Geografia. Rio de Janeiro - RJ, v. 57, n. 1, jan/mar, 1995.

PARAÍBA. Atualização Covid-19. João Pessoa - PB: Sala de Imprensa da Secretaria Estadual de Saúde - PB, 2020. Disponível em: https://paraiba.pb.gov.br/diretas/saude/coronavirus/noticias. Acesso em: 05 de maio de 2020.

WHO. Coronavirus disease (COVID-19): situation report. Genebra, 2020. Disponível em: https://www.who.int/emergencies/diseases/novel-coronavirus-2019/situation-reports/. Acesso em 01 de maio de 2020.

WOO, LAU, CHU, et al. Characterization and complete genome sequence of a novel coronavirus, coronavirus HKU1, from patients with Pneumonia. Journal of Virology, USA, American Society for Microbiology. P. 884-895, jan 2005. Disponível em: https://jvi.asm.org/content/79/2/884.full. Acesso em 02 mai 2020. https://doi.org/10.1128/JVI.79.2.884-895.2005 